\title{
Performance assessment of clustering algorithms for the thermal testing of industrial components.
}

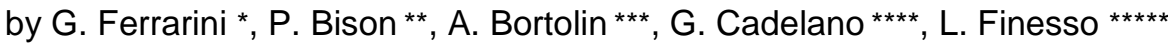

\author{
*CNR-ITC, Corso Stati Uniti 4, 35127 Padova PD, Italy, giovanni.ferrarini@itc.cnr.it \\ ** CNR-ITC, Corso Stati Uniti 4, 35127 Padova PD, Italy, paolo.bison@itc.cnr.it \\ *** CNR-ITC, Corso Stati Uniti 4, 35127 Padova PD, Italy, alessandro.bortolin@itc.cnr.it \\ **** CNR-ISAC, Corso Stati Uniti 4, 35127 Padova PD, Italy, g.cadelano@isac.cnr.it

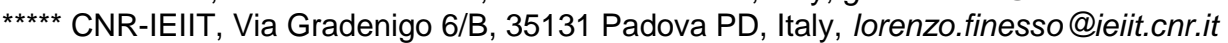

\begin{abstract}
Infrared thermography is a widely applied technique for the thermal testing of industrial components. The thermal data obtained with an infrared test are processed with different algorithms to improve the detection of defects. This work investigates the performance of the clustering algorithms to define the defective and non-defective regions of a steel specimen, with flat bottom-hole defects, inspected with a flash thermography setup. A clustering algorithm with different parameters is used to analyze the thermal data coming from the experiment, assessing the performance of the algorithm in terms of defect detection.
\end{abstract}

\section{Introduction}

Infrared thermography is currently a standard method for the thermal inspection of industrial components [1,2] with a wide range of applications, including metallic and composite materials. The typical experiment layout consists in a thermal camera that records the temperature evolution of a specimen when a thermal stimulus (e.g. flash, sinusoidal heating) is applied on it. The obtained temperature data are then analyzed with different image processing algorithms, to help the operator discerning the defective regions from the sound ones. Relevant advances in the detectability of defects have been made in the recent times, thanks both to the improvement of the thermal sensors and to the introduction or refinement of data analysis methods. In the scientific literature, an extremely wide range of data processing algorithms is available and the thermographic operator should choose the most effective one for each kind of inspected specimen and testing procedure.

This work is focused on the use of the k-means clustering algorithm as the processing method of thermal sequences. The k-means [3] is a well-known algorithm that is applied in different fields such as biology, medicine, market analysis. The goal of the algorithm is to divide the data in groups, called clusters, that are meaningful, useful, or both [4]. The use of cluster analysis in infrared thermography has been proposed for the classification of thermal anomalies [5,6], trying to obtain defective and non-defective regions. In this work, different analysis procedures based on the clustering algorithm are compared in terms of defect detection performance.

\section{Experimental procedure}

Firstly, a set of thermographic data is obtained from the inspection of a steel specimen with flat bottom-hole defects. The experimental layout is depicted in the following figure 1.

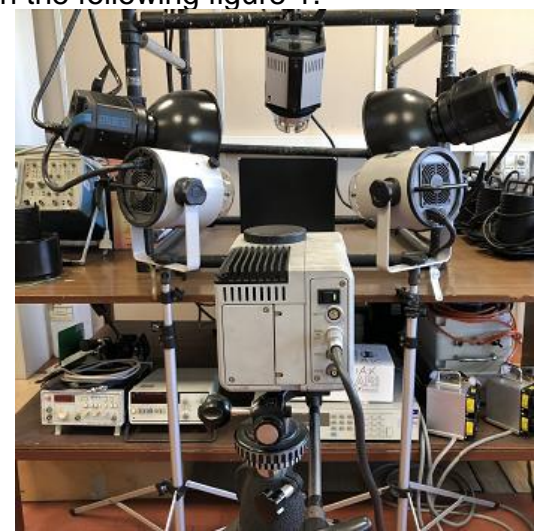

Fig. 1. Experimental layout. The thermal camera is pointed at the specimen which is thermally stimulated by a set of lamps. 
The thermal stimulus is a flash provided by 5 lamps, summing up to around $14 \mathrm{~kJ}$ of electrical power. The thermal camera has a quantum well sensor (spectral response between 8.5 and $9.5 \mu \mathrm{m}$ ) with a resolution of 320 by 240 pixels.

The specimen has 16 circular defects with different diameters and depths. The defect map is shown in the following figure 2 .

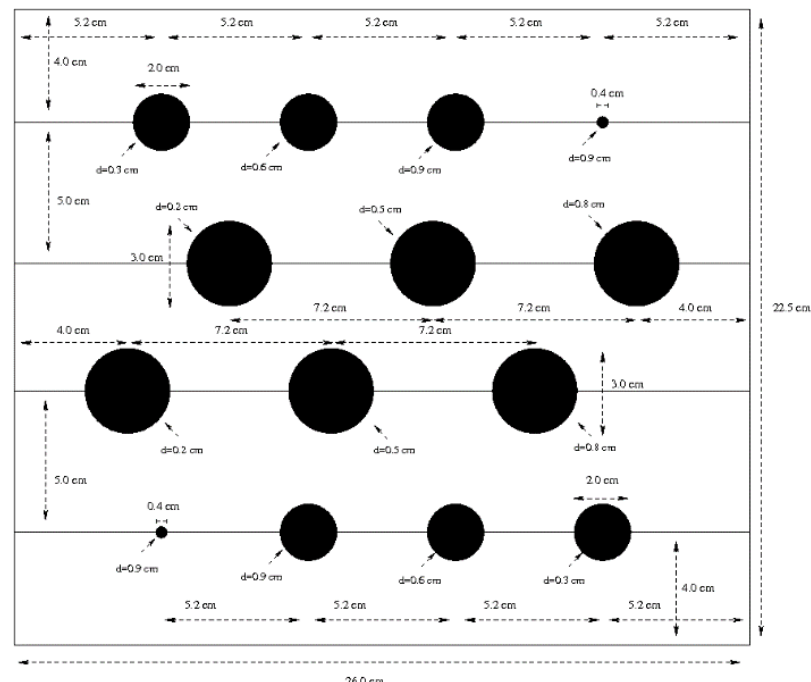

Fig. 2. Defect map of the tested specimen.

The data obtained from the inspection are either kept raw or pre-processed with filtering and de-noising algorithms. On both kinds of data, the clustering algorithm is applied. After the initial selection of k points as the initial centroids, the procedure consists in assigning each data point to its closest centroid (forming $\mathrm{k}$ clusters) and then recomputing the centroid of each cluster. The last steps are repeated until the centroids do not change anymore [4]. The performance of the algorithm in defect classification is then evaluated with the receiving operator characteristic (ROC) curves [7]. The performance is influenced by different factors as the choice of the proximity function, of the number of clusters, of the location of the centroids, of the pre-processing algorithm.

\section{REFERENCES}

[1] D. Balageas, X. Maldague, D. Burleigh, V.P. Vavilov, B. Oswald-Tranta, J.-M. Roche, C. Pradere, G.M. Carlomagno, Thermal (IR) and Other NDT Techniques for Improved Material Inspection, J. Nondestruct. Eval. 35 (2016) 18. https://doi.org/10.1007/s10921-015-0331-7.

[2] X. Maldague, Theory and practice of infrared technology for nondestructive testing, Wiley, 2001.

[3] D. Steinley, K-means clustering: A half-century synthesis, Br. J. Math. Stat. Psychol. 59 (2006) 1-34. https://doi.org/10.1348/000711005X48266.

[4] P.-N. Tan, M. Steinbach, A. Karpatne, V. Kumar, Introduction to Data Mining, 2 edition, Pearson, NY NY, 2018.

[5] R. Vishnupriya, N.S.M. Raja, V. Rajinikanth, An efficient clustering technique and analysis of infrared thermograms, in: 2017 Third Int. Conf. Biosignals Images Instrum. ICBSII, 2017: pp. 1-5. https://doi.org/10.1109/ICBSII.2017.8082275.

[6] K. Zheng, Y.-S. Chang, K.-H. Wang, Y. Yao, Thermographic clustering analysis for defect detection in CFRP structures, Polym. Test. 49 (2016) 73-81. https://doi.org/10.1016/j.polymertesting.2015.11.009.

[7] L. Lei, G. Ferrarini, A. Bortolin, G. Cadelano, P. Bison, X. Maldague, Thermography is cool: Defect detection using liquid nitrogen as a stimulus, NDT E Int. 102 (2019) 137-143. https://doi.org/10.1016/j.ndteint.2018.11.012. 